This is an electronic reprint of the original article. This reprint may differ from the original in pagination and typographic detail.

\author{
Author(s): Takalo, Jouni; Timonen, Jussi; Sampo, Jouni; Rantala, Maaria; Siltanen, Samuli; Lassas, \\ Matti
}

Title: $\quad$ Towards a "fingerprint" of paper network; separating forgeries from genuine by the properties of fibre structure

Year: $\quad 2014$

Version:

Please cite the original version:

Takalo, J., Timonen, J., Sampo, J., Rantala, M., Siltanen, S., \& Lassas, M. (2014).

Towards a "fingerprint" of paper network; separating forgeries from genuine by the properties of fibre structure. In D. Burgess, G. Owen, H. Rana, R. Zamboni, F. Kajzar, \& A. A. Szep (Eds.), Optics and Photonics for Counterterrorism, Crime Fighting, and Defence $X$; and Optical Materials and Biomaterials in Security and Defence Systems Technology XI (Article 92530A). SPIE - International Society for Optical Engineering. SPIE conference proceedings, 9253. https://doi.org/10.1117/12.2066809

All material supplied via JYX is protected by copyright and other intellectual property rights, and duplication or sale of all or part of any of the repository collections is not permitted, except that material may be duplicated by you for your research use or educational purposes in electronic or print form. You must obtain permission for any other use. Electronic or print copies may not be offered, whether for sale or otherwise to anyone who is not an authorised user. 


\title{
Towards a "fingerprint" of paper network; separating forgeries from genuine by the properties of fibre structure
}

Jouni Takalo (1), Jussi Timonen (1,2,3), Jouni Sampo (4,5), Maaria Rantala (5), Samuli Siltanen (5), and

Matti Lassas (5)

(1) Department of Physics, University of Jyväskylä, Finland, email: jouni.j.takalo@jyu.fi (2) Nanoscience Center, University of Jyväskylä, Finland (3) ITMO University, Kronverkskii ave. 49, 197101, Saint Petersburg, Russia (4) Department of Mathematics and Statistics, Technical University of Lappeenranta, Finland (5)

Department of Mathematics and Statistics, University of Helsinki, Finland

\begin{abstract}
A novel method is introduced for distinguishing counterfeit banknotes from genuine samples. The method is based on analyzing differences in the networks of paper fibers. The main tool is a curvelet-based algorithm for measuring the distribution of overall fiber orientation and quantifying its anisotropy. The use of a couple or more appropriate parameters makes it possible to distinguish forgeries from genuine samples as concentrated point clouds in a two- or three-dimensional parameter space. Furthermore, the techniques of making watermarks is investigated by comparing genuine and counterfeit $€ 50$ banknotes. In addition, the so-called wire markings are shown to differ significantly from each other in all of investigated banknotes.
\end{abstract}

KEYWORDS: forensic science, counterfeit banknotes, fiber network, fiber orientation, curvelet 


\section{INTRODUCTION}

Forgeries and fakes are made all over: paintings, banknotes, stamps, and other valuable papers among other things. Traditionally forgeries have been distinguished by visual inspection or by machines that can identify specific security markers inserted in the paper. These include watermarks, fluorescent threads that can be seen only in ultraviolet light, security strips, and holograms. However, while forgery is made more difficult, counterfeiters follow suit and learn to better mimic these security markers.

We propose a novel method for analyzing the characteristics of the fiber network of paper products. The method is based on processing optical transmission images of paper samples using an orientation-sensitive algorithm called curvelet transform. Furthermore, we use some additional parameters to characterize the paper structure. Extracting two, three, or more representative parameters from optical and x-ray images leads to a 'fingerprint' of the fiber network. Forgeries can be discerned from genuine samples by their fingerprints based solely on the properties of the underlying paper.

In addition to numerous small-scale counterfeits, in some cases governments need to be prepared for highquality counterfeit currency in large scale. A well-known example of such a case happened during World War II when Germany aimed to use counterfeiting as a weapon and forged British pounds. ${ }^{1}$ More recently, there have appeared high-quality counterfeits of US dollars, called 'superdollars' or 'supernotes', because of their high similarity to the real US dollar. ${ }^{9,14}$ Countermeasures against such high-quality counterfeits require swift actions, e.g., the US Treasury introduced in 1996 a new $\$ 100$ banknote so as to decrease such counterfeiting. ${ }^{11}$

The present study is mostly based on measuring optical parameters of paper, and we utilize a special type of wavelets called curvelets in the analysis of optical images. Furthermore, we use x-ray transmission images so as to characterize so-called wire marking in the paper structure ${ }^{17}$ and compare techniques of making a watermark in banknotes. Our main goal is to provide a set of parameters determined by precise methods, which would charaterize a given paper network so that the papers used to print genuine and counterfeit documents can be told apart. The final goal is to find in this way a 'fingerprint' of basically each individual paper. To this end we concentrate here on $€ 50$ banknotes, and introduce different methods for characterization of the respective paper substrates. Also, these methods may be applied to help classify and choose the paper substrate before printing the document in question.

Earlier studies on genuine and counterfeit banknotes have been done using numerous methods, e.g., wavelets, ${ }^{7}$ infrared spectroscopy ${ }^{20}{ }^{20}$ ass spectrometry, ${ }^{10}$ optical-coherence tomography, ${ }^{8}$ Raman microspectroscopy, ${ }^{15}$ Mössbauer spectroscopy, ${ }^{18}$ supporting vector machines, ${ }^{21}$ neural networks, ${ }^{13}$ and measuring the lifetime of fluorescence in banknotes. ${ }^{6}$ The novelty of the present investigation is that it focuses on the underlying paper structure, which, according to our knowledge, has not been done in this context before.

\section{MATERIALS AND METHODS}

\subsection{The optical measurement system}

Optical measurements of a sample was done by setting it on an illuminated table, and recording the transmitted light directly above it so as to get a so-called bright-field image. A reflected-light image was taken using a socalled cold light source which was set in an inclination angle of about 20 degrees between the light direction and the surface of the banknote. Images were recorded with a Canon EOS 600D camera mounted to a microscope.

\subsection{Determination of orientation}

For the evaluation of fiber orientation we used the method recently introduced by Sampo et al., ${ }^{19}$ which is based on directional wavelets, so-called curvelets. ${ }^{3,4}$ It has been shown that curvelet-based orientation analysis is, in most cases, better than those made by more traditional methods, like the gradient-based structure-tensor (ST) method $^{2,16}$ or direct FFT-based ${ }^{12}$ methods. Curvelet-based orientation analysis is more robust, in particular when the resolution of the image decreases. ${ }^{19}$ We have also shown that the curvelet method is robust and rather insensitive to noise in the image. This property increases its usability in practical applications. For clarity, we give a short desscription of this method here. 

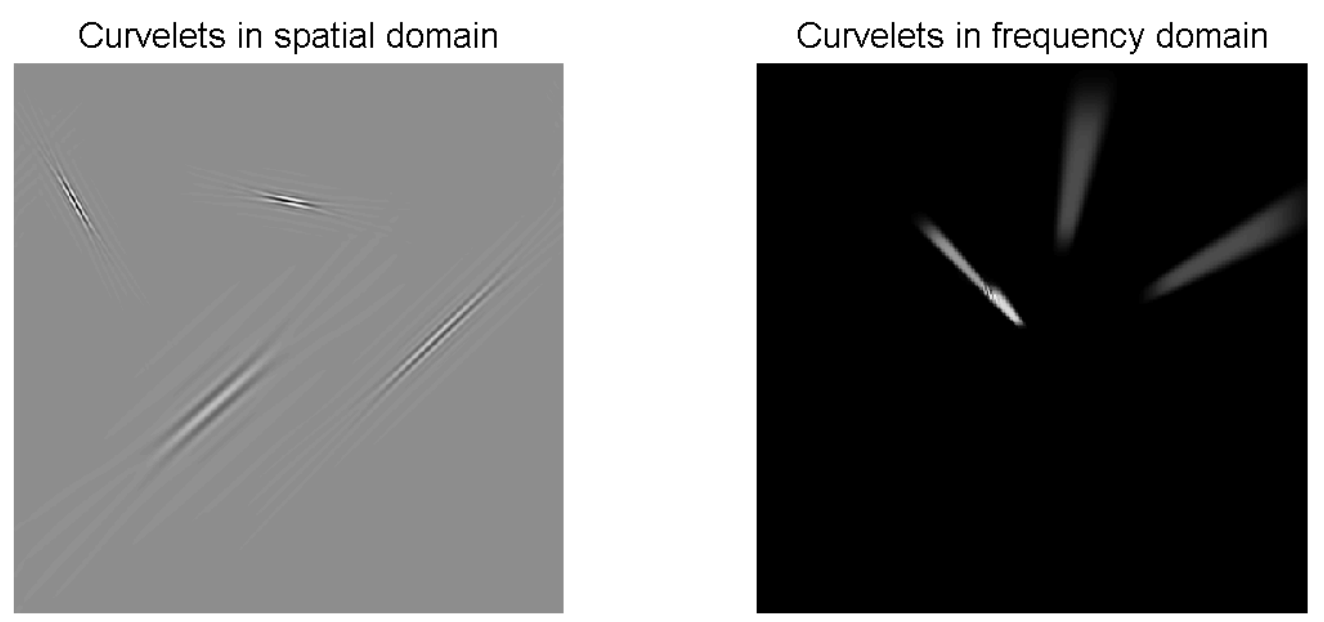

Figure 1. Four curvelets with different orientation, location, and scale. This image was produced with CurveLab. ${ }^{5}$

Let $f$ be a representation of a grey scale (optical) image of the sample. We can express this representation in terms of basis functions $\phi_{b}$ and $\gamma_{a b \theta}$ such that

$$
f=\sum_{b}\left\langle f, \phi_{b}\right\rangle \phi_{b}+\sum_{a, b, \theta}\left\langle f, \gamma_{a b \theta}\right\rangle \gamma_{a b \theta} .
$$

Functions $\phi_{b}$ are restricted to low frequencies and are therefore not relevant in this application. However, functions $\gamma_{a b \theta}$ are called curvelets. They have discretizations for the scale $(a)$, location $(b)$, and angle $(\theta)$, which are approximately like $a_{j}=2^{-j}, j=1,2, \ldots ; \theta_{j, l}=2 \pi \cdot l \cdot 2^{-\lfloor j / 2\rfloor}, l=0,1, \ldots, 2^{\lfloor j / 2\rfloor}-1 ;$ and $b_{k}^{(j, l)}=$ $R_{\theta_{j, l}}\left(k_{1} 2^{-j}, k_{2} 2^{-j / 2}\right), k_{1}, k_{2} \in Z$. $^{3}$ In Fig. 1 four curvelet functions $\gamma_{a b \theta}$ are illustrated in both the spatial and frequency domain (zero frequency in the middle). The curvelets of the two largest scales have the same orientation, while the two curvelets of smaller scale have orientations that are different from that of the other two. In the frequency domain curvelets are smooth and compactly supported, while in the spatial domain they decay rapidly in the dominating dimension (along the long axis of its elongated shape) and oscillate in the perpendicular direction. The important difference to ordinary wavelets is that curvelets obey parabolic scaling, which means that their width-to-length ratio is $a^{1 / 2}$, while for wavelets this ratio is independent of scale. Another difference to the regular wavelet transforms is that for curvelets, the number of orientations grows when the scale decreases. These features make curvelets very efficient for approximating or analyzing edges. ${ }^{4}$

In small scales $a$, the magnitudes of inner products $\left\langle f, \gamma_{a b \theta}\right\rangle$ are large for such curvelets $\gamma_{a b \theta}$ that are oriented like edges in an image. ${ }^{19}$ Moreover, curvelets form a tight frame, i.e., $\|f\|_{2}^{2}=\sum_{b}\left|\left\langle f, \phi_{b}\right\rangle\right|^{2}+\sum_{a, b, \theta}\left|\left\langle f, \gamma_{a b \theta}\right\rangle\right|^{2} .4$ This property suggests that an estimate for the orientation distribution could be expressed in the form

$$
S(\theta):=\frac{\sum_{a \in I} \sum_{b \in J_{a}}\left|\left\langle f, \gamma_{a b \theta}\right\rangle\right|^{2}}{\int_{0}^{\pi} \sum_{a \in I} \sum_{b \in J_{a}}\left|\left\langle f, \gamma_{a b \theta}\right\rangle\right|^{2} d \theta},
$$

where the index set $I$ for the scales depends on the resolution and size of the particles in the image, such that we do not capture any unwanted structures. In this work we used the two smallest scales depending on the

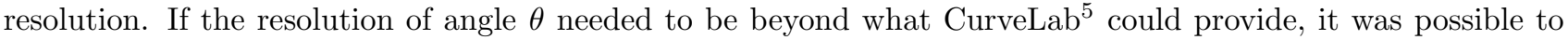
generate any $\gamma_{a b \theta}$ by rotating $\gamma_{a b 0}$. For each scale we had a rectangular grid of points as the translation set,

$$
J_{a}=\left\{R_{\theta}\left(C_{1} l a, C_{2} k a^{1 / 2}\right)^{T}:(l, k) \in Z^{2}\right\},
$$

with constanst $C_{1}$ and $C_{2} \cdot{ }^{19}$ 


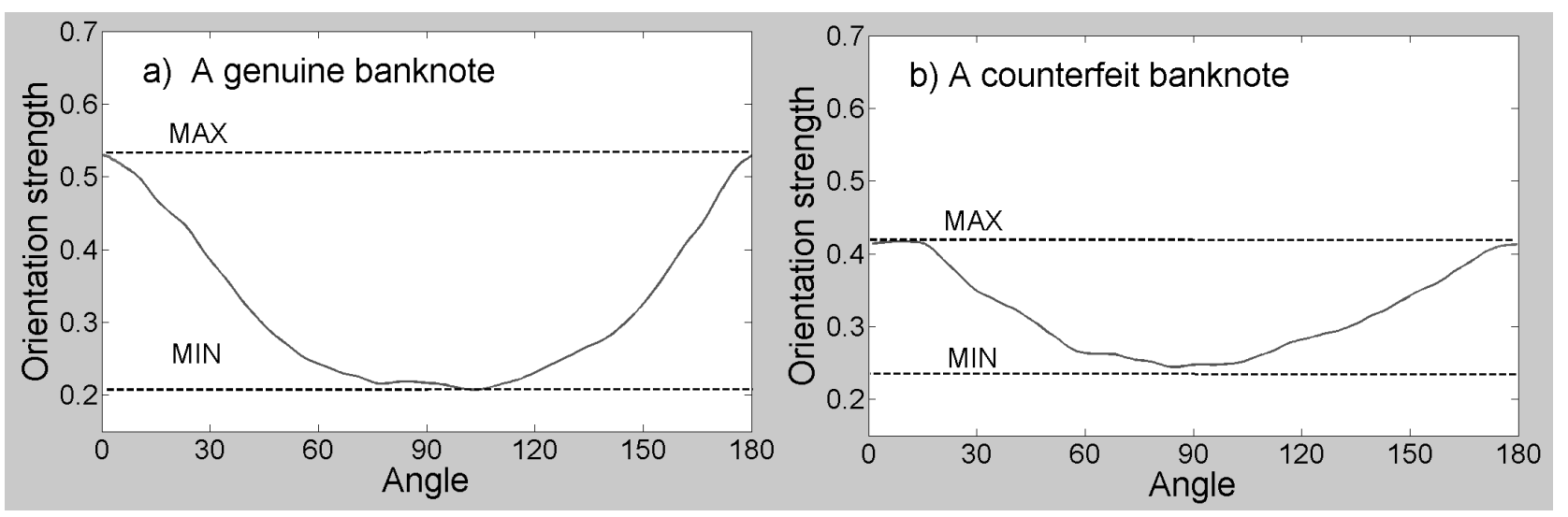

Figure 2. Examples of orientation distribution of fibers for (a) a genuine banknote and (b) a counterfeit banknote. The maximum and minimum of the distribution used to define the anisotropy (as their ratio) are shown by dotted horizontal lines.

\subsection{Material of analysis}

In the analysis of banknotes we used ten counterfeit $€ 50$ banknotes, and two different series of 30 unused genuine $€ 50$ banknotes. These two series were printed on paper substrates provided apparently by two different manufacturers. The counterfeit banknotes were provided by the Finnish National Bureau of Investigation, and the genuine banknotes by the Bank of Finland.

\section{ANALYSIS AND RESULTS}

\subsection{Parameter space}

In order to discern forgeries from genuine banknotes, we needed to find parameters that separate different fiber structures. Since the above-mentioned orientation analysis proved to be good, a natural parameter of characterization was the main orientation direction of the fibers. The main orientation of fibers in a paper (machine) is usually near the so-called machine direction (MD) ${ }^{17}$ which is the running direction of the machine. The minimum of the orientation distribution is then usually in a direction perpendicular to that direction, in the so-called cross direction (CD). We show examples of orientation distributions of one genuine banknote in Fig. 2a, and the orientation distribution of a counterfeit banknote in Fig. 2b. Distributions were normalized so as to have an area of one between the distribution curve and the angle axis (in radians). Another parameter we get from the orientation analysis is the anisotropy of the distribution, ${ }^{17}$ which we define here simply as the ratio of distribution maximum to distribution minimum of the normalized distribution (see dotted lines in Fig.2). As an additional parameter for banknotes we used the pinhole (a small hole in the paper structure) percentage, which in a sense describes how sparse the underlying paper is. This parameter was measured from binary images as shown in Fig. 3 for a genuine and a forged banknote.

In banknote analysis we used ten $€ 50$ counterfeit banknotes, which were provided by the Finnish National Bureau of Investigation. There were three different counterfeits: Two with serial number A (for security reasons we do not use the exact ID), three with ID B and five with ID C. As a reference of a genuine set of banknotes we used 30 pieces of two different series of notes with identification numbers starting with letters X (Germany) and $\mathrm{Z}$ (Belgium). The analyzed images were taken from the region of the European union flag, because the blue color gives a good contrast for the visibility of fibers (see Fig. 4). Because both genuine and counterfeit banknotes were made recently, fibers turned out to be of a similar thickness, and we could not use it as a separating parameter. The anisotropy of the orientation, however, changed even between the different series of the genuine banknotes. The paper grade of the counterfeits was also inferior to the pure cotton paper of the genuine banknotes, and the so-called pinholes (where light passes directly trough the paper) were more abundant in the counterfeit banknotes. That is why we defined a parameter that we call a pinhole percentage, for description of 'porosity' of the paper grade. Each of the grey-scale images was adjusted to have about the same mean of the histogram. However, the counterfeits had more skewed histograms and thus slightly larger standard deviations. A typical 

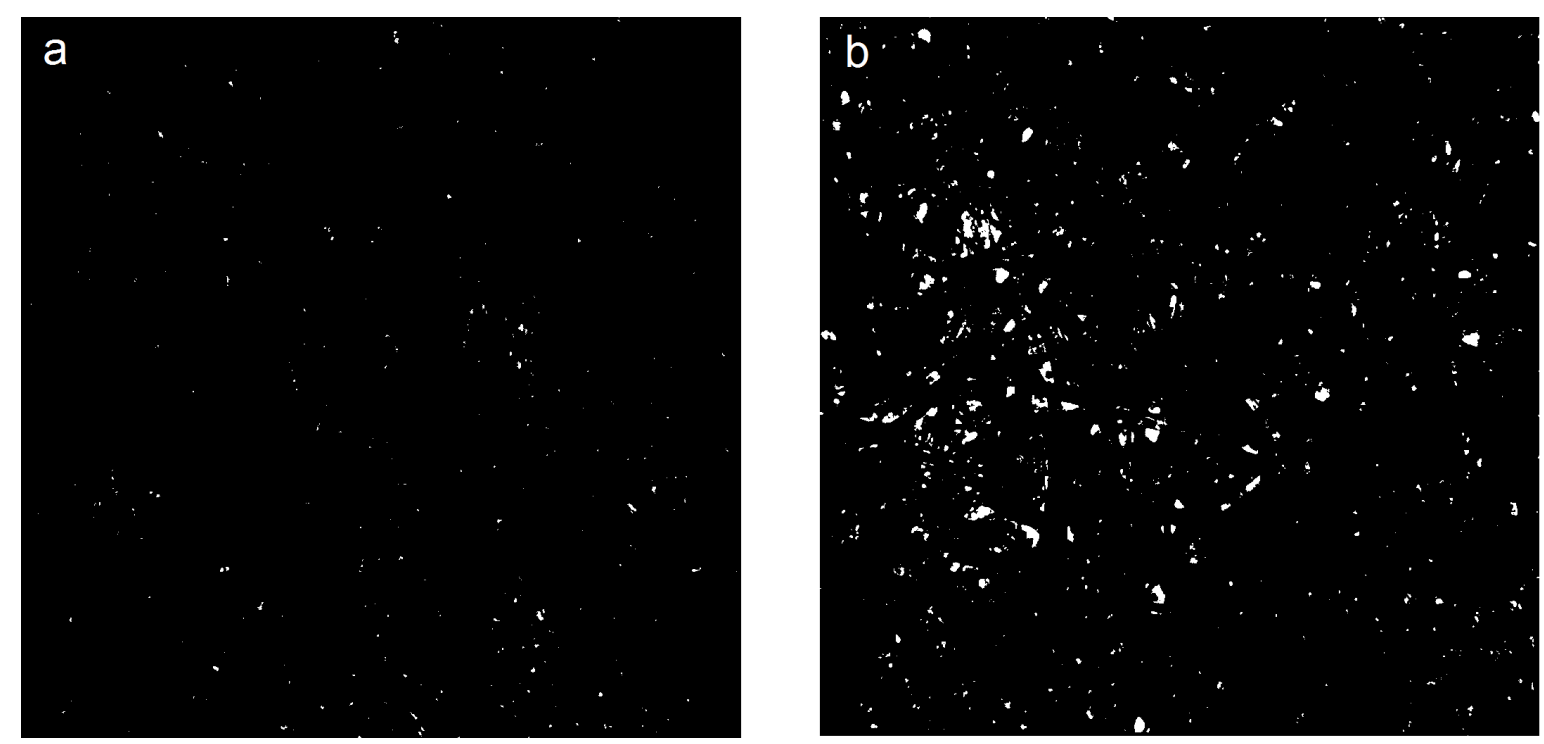

Figure 3. a) Pinholes in a binary image of a genuine $€ 50$ banknote. b) Pinholes in a binary image of a counterfeit $€ 50$ banknote.

binary figure using a threshold value of 244 (grey-scale values were in the range 0-255) for binarization is depicted in Fig. 3 that shows about ten times more pinhole area in the counterfeit than in the genuine banknote. As a reference for the genuine banknotes, we analyzed all sixty 50-euro banknotes of the X- and Z-series, and show their locations in a 3D parameter space in Fig. 5. It is evident that the Z-series banknotes are more compactly located in that parameter space than the X-series banknotes.

Most of the counterfeits also had the main orientation in the direction of the shorter side of the banknote, which is clearly the main orientation direction of all genuine banknotes analyzed. That is why we omitted the orientation direction from the parameters and show the counterfeit banknotes only in a 2D parameter space with anisotropy and pinhole percentage as the parameters (see Fig. 6). We show there also the results for all genuine banknotes. It is evident that the counterfeit banknotes are separated from the genuine ones independent of whether they are from the X- or Z-series. Also the two sets of genuine banknotes can be separated with these two parameters. This is probably because they had apparently been printed on paper substrates provided by different manufacturers. The mean value of anisotropy for the $\mathrm{X}$-series banknotes was $1.80+/-0.23$, while it was $2.32+/-0.15$ for the $\mathrm{Z}$ series (mean values shown as squares in Fig. 6). On the other hand, the X-series had a smaller amount of pinholes, although both X- and Z-series banknotes had only about a tenth part of what the counterfeit banknotes.

\subsection{Watermarks}

One of the oldest security marks to ensure the authenticity of banknotes and other valuable documents has been watermark. However, watermarks are quite easy to mimic, at least, for verification by visual inspection. Here we compare the techniques used for making watermarks in genuine and some counterfeit banknotes. In the Fig. 7 we depict the images in the reflected (7a) and transmitted light (7b) of a genuine Z-series $€ 50$ banknote. The inclination angle (angle between the surface of the banknote and the source of light) was quite small to show the topography of the watermark. Notice that in the genuine banknote watermark is done by thinning the paper at the sites, where the intensity of the transmitted light was bigger. In Fig. 8 we show two examples of counterfeit $€ 50$ banknotes with images of the watermarks in a reflected light (Figs 8a and c), and in a transmitted light (Figs $8 \mathrm{~b}$ and $\mathrm{d}$ ). These images reveal that the counterfeit watermarks have been pressed with a some kind of raster on the underlying paper such that there is more fiber mass in the area, where the intensity of transmitted light should be smaller. 


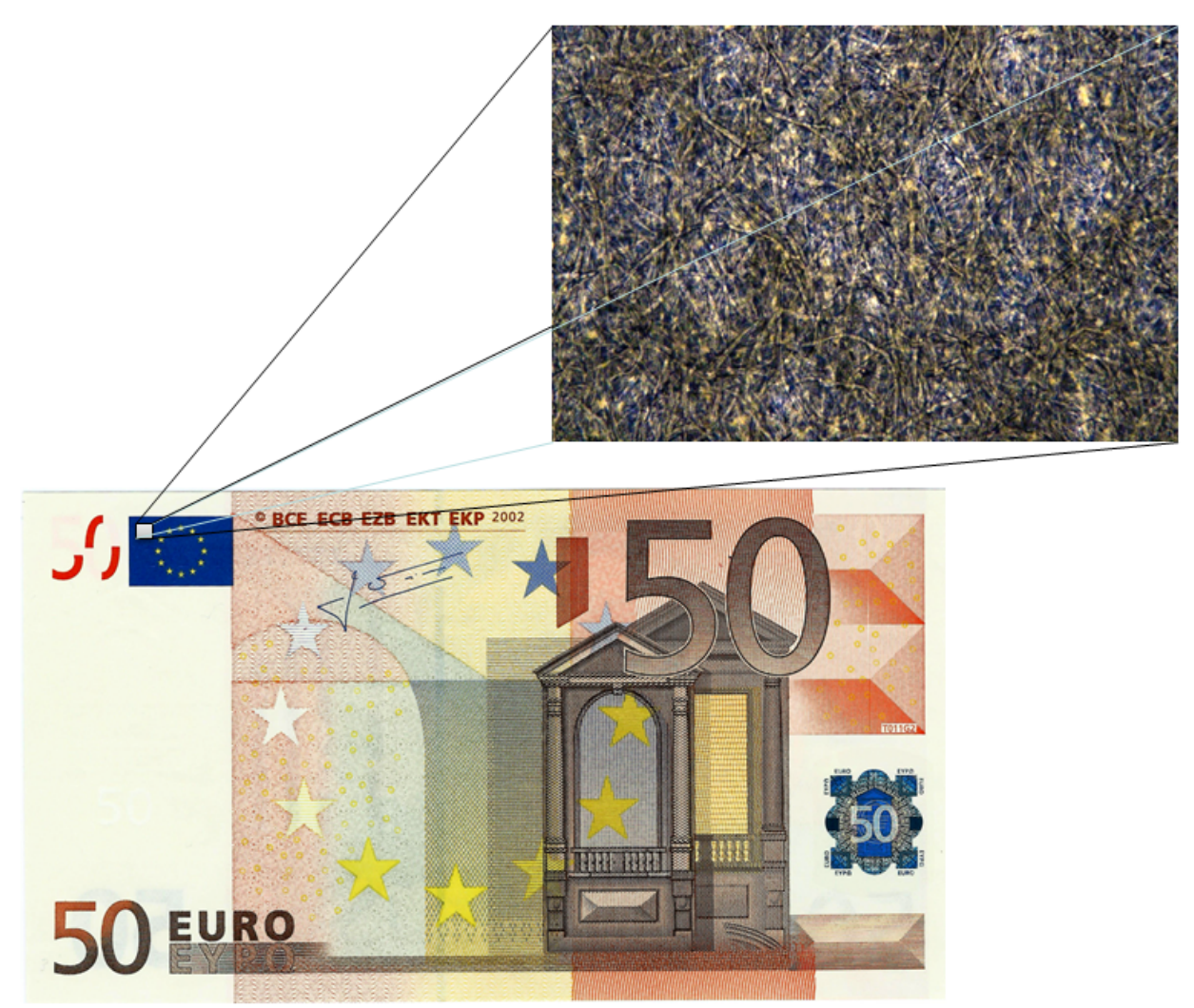

Figure 4. An $€ 50$ banknote and magnification of the area of the European union flag from where the fiber-image samples of the banknotes were taken. The real area of the sample is $1.9 \mathrm{~mm} \bullet 2.9 \mathrm{~mm}$

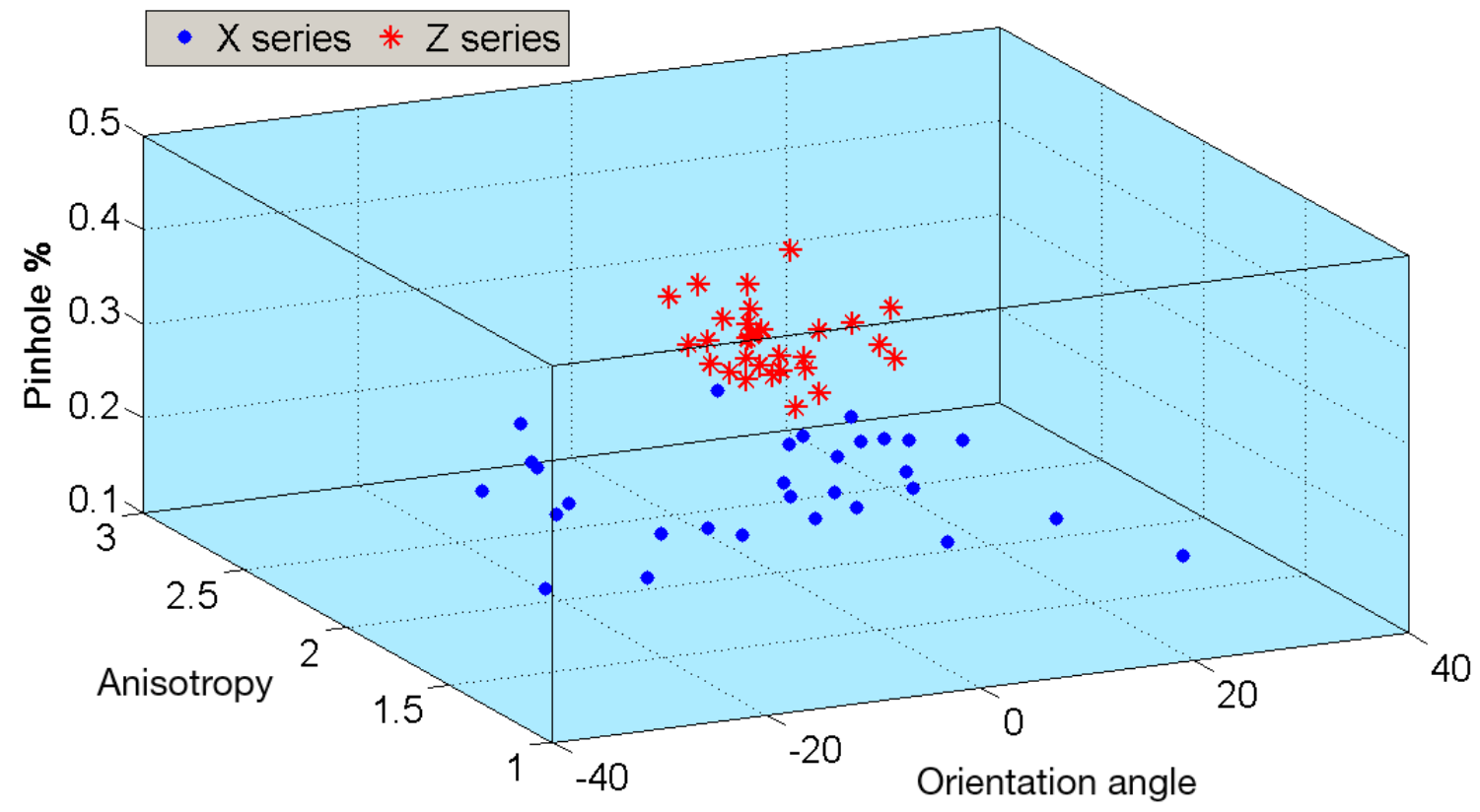

Figure 5. Results for all genuine banknotes shown in a 3D parameter space (with blue dots for the X series and with red stars for the $\mathrm{Z}$ series). 


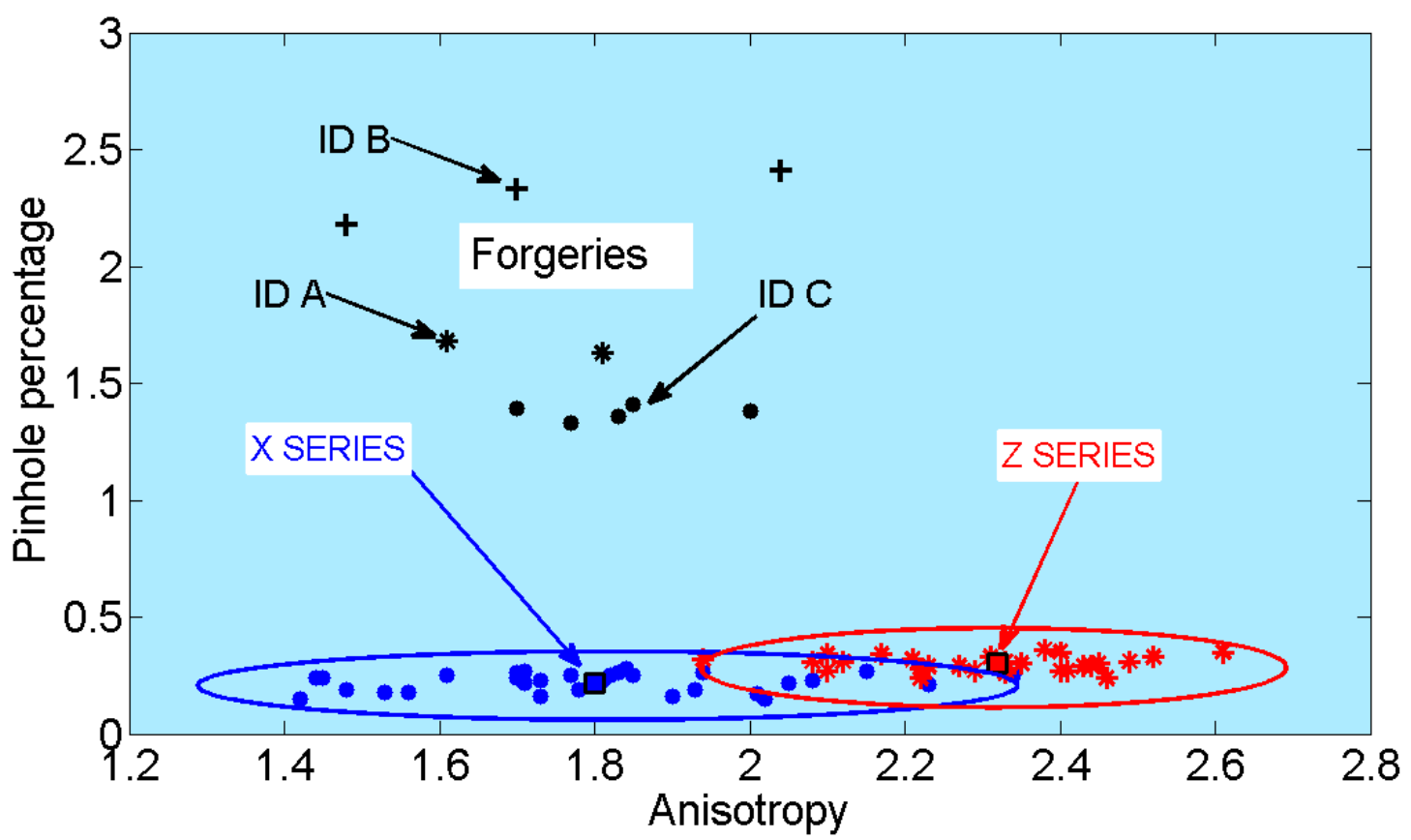

Figure 6. Results for two thirty-piece sets of genuine $€ 50$ banknotes and for ten counterfeit $€ 50$ banknotes in a $2 \mathrm{D}$ parameter space. Mean values of the genuine banknotes are shown as colored squares.
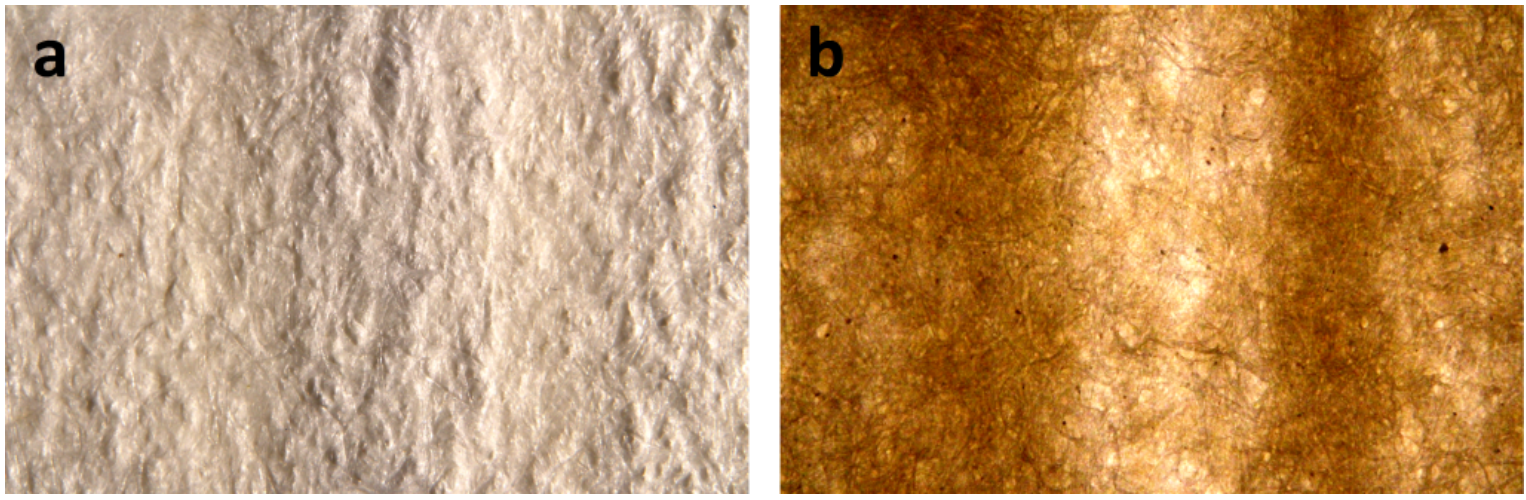

Figure 7. a) An image of the watermark of a genuine $€ 50$ banknote in an inclined reflected light. b) A transmission image of the same area of the watermark. 

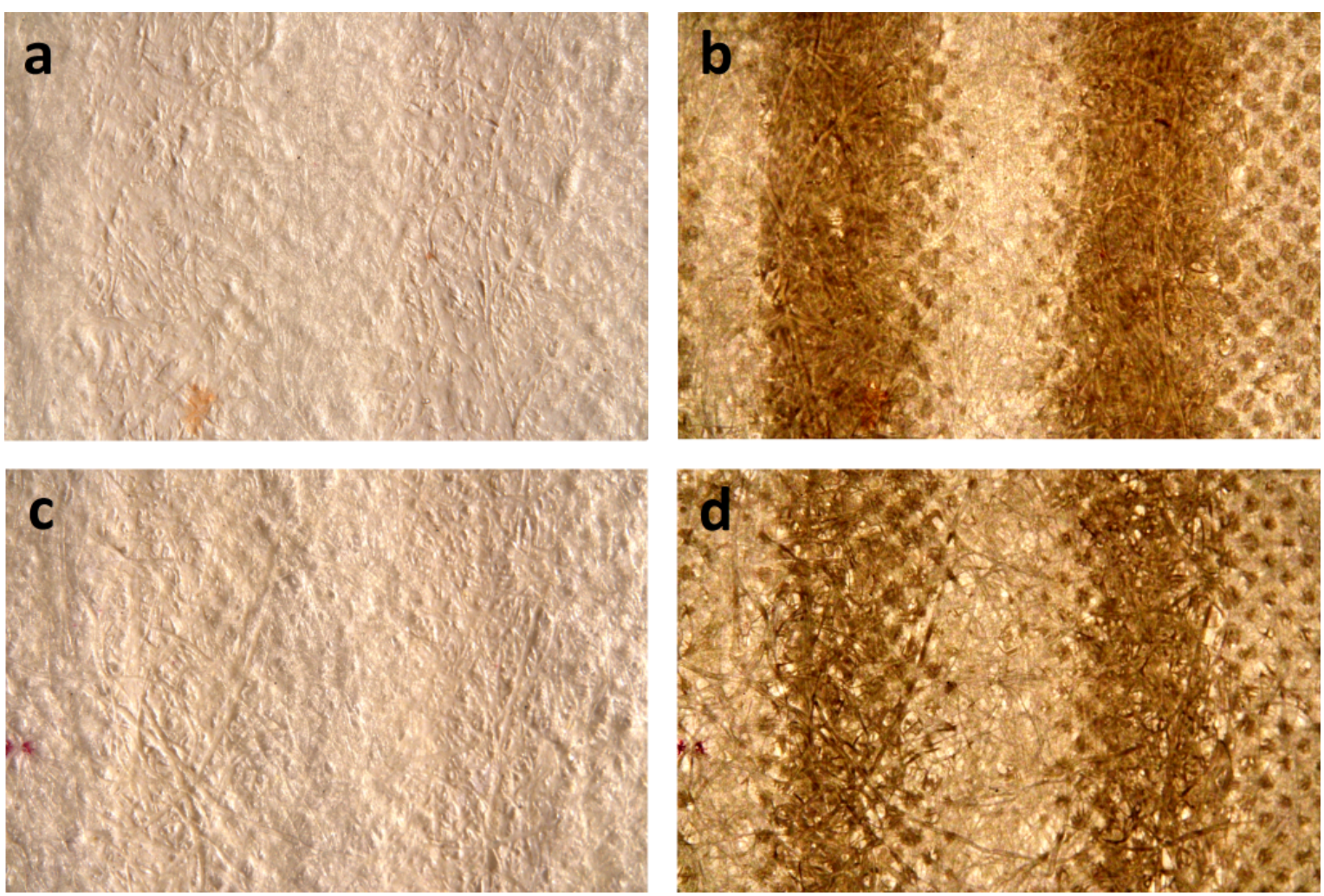

Figure 8. a) and c) Watermarks of two counterfeit $€ 50$ banknotes in an inclined reflected light. b) and d) Transmission-light images of the same areas of the watermarks.

\subsection{Wire marking}

We then took x-ray transmission images of several genuine and counterfeit banknotes. Although these banknotes were full of safety markings, we found a region of about $1 \mathrm{~cm}^{2}$ with no safety features, which was then used in this analysis. After wavelet denoising, marks of the so-called pressing wire of the paper machine were quite visible in these bright-field images as is evident from Fig. 9, where such images are shown for a sample of the Z series (panel a), a sample of the X series (panel b), and for two samples of the counterfeit banknotes (panels c and $\mathrm{d}$ ). It is evident that the wires used in the manufacturing of the $\mathrm{X}$-series and Z-series banknotes had been different. On the other hand, the wire markings in the counterfeit banknotes were totally different from those in the genuine banknotes. Wire marking is, in a sense, a signature of the paper machine used, and it is thus a useful parameter in detecting the origin of the paper used in any document.

\section{DISCUSSION}

We reported here a new method for identifying counterfeit banknotes, which was based on analyzing differences in the structure of the paper substrates used in them. To this end we applied a number of different parameters for characterization of that structure, but this type of analysis is not restricted to these parameters alone. We found particularly useful the orientation distribution of the fibers in paper, for which we used our recently developed curvelet-based method. From this distribution we used here the main orientation direction and the anisotropy of orientation as the specific parameters. We also found the pinhole percentage very useful. This parameter describes the amount of small holes in paper and is related to how sparse (or heterogeneous) the structure is. With these parameters we showed that it is possible to separate forgeries from genuine samples in a $2 \mathrm{D}$ or $3 \mathrm{D}$ parameter space. Even different series of genuine banknotes could be distinguished from each other using the wire marking typical of an individual paper machine.

Furthermore, we distinguished the counterfeit banknotes from genuine ones by differences in their watermarks and from wire markings which are charateristic of the paper machine used. Although we had here banknotes as 

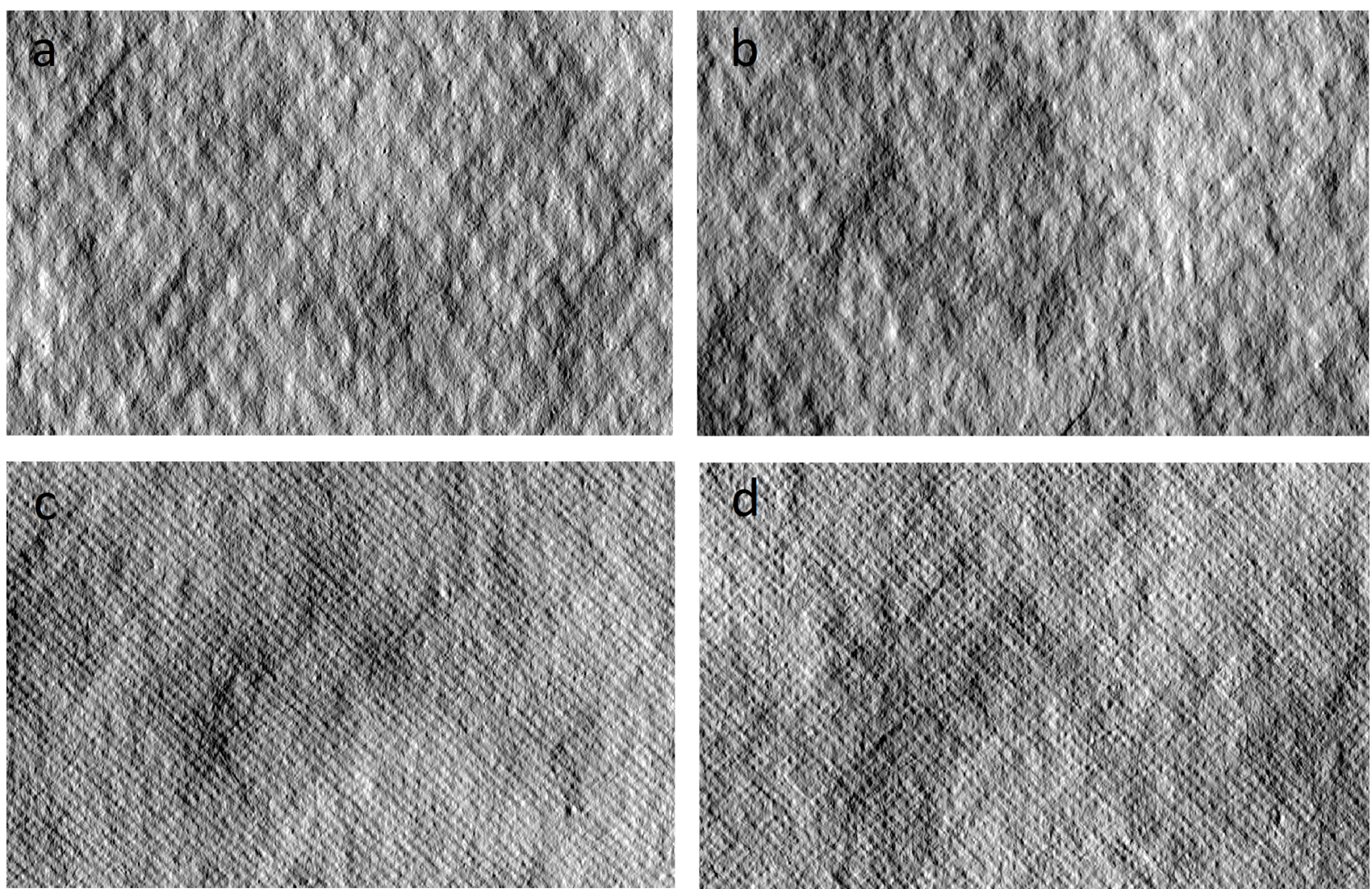

Figure 9. X-ray transmission images (after denoising) of the paper used in a) the genuine banknotes of the $\mathrm{Z}$ series, b) the genuine banknotes of the $\mathrm{X}$ series, c) the ID A counterfeit banknotes, and $\mathrm{d}$ ) the ID $\mathrm{C}$ counterfeit banknotes. The real area of each figure is about $12.3 \cdot 7.5 \mathrm{~mm}^{2}$.

an example, we believe that a similar method can be applied to investigation of other value documents. Our final goal is to define a 'fingerprint' of paper by which each individual set (manufactured by the same paper machine) of samples can be recognized.

\section{Acknowledgement}

We are grateful to the Academy of Finland (projects no. 255891, 255824 and 250215) and Forest Cluster Ltd for financial support. We also thank the Finnish National Bureau of Investigation and the Bank of Finland for providing part of the material of this study.

\section{REFERENCES}

1. L. Allen, The Encyclopedia of Money, 2nd edition, ABC-CLIO, LLC, Santa Barbara, California, US, 2009.

2. T. Brox, R. van den Boomgaard, F. Lauze, J. van de Weijer, J. Weickert, P. Mrzek, P. Kornprobst, Adaptive structure tensors and their applications, in: J. Weickert, H. Hagen (Eds.), Visualization and Processing of Tensor Fields, Springer-Verlag, Berlin, pp. 17-47, 2006.

3. E.J. Candès, What is a curvelet?, Notices Amer. Math. Soc., 50, no. 11, 1402-1403, 2003.

4. E.J. Candès and D. L. Donoho, New tight frames of curvelets and optimal representations of objects with piecewise $C^{2}$ singularities, Commun. Pure Appl. Math., 57, no. 2, 219-266, 2004.

5. E.J. Candès, L. Demanet, D. L. Donoho, and L. Ying, Fast Discrete Curvelet Transforms, Multiscale Model. Simul., 5(3), 861-899. 2006.

6. T. H. Chia, and M. J. Levene, Detection of counterfeit U.S. paper money using intrinsic fluorescence lifetime, Optics Express, vol 17, no. 24, pp. 22054-22061, 2009. 
7. Fuisun Choi, Jongseok Lee, and Joonhyun Yoon, Extraction for Bank Note Classification Using Wavelet Transform, ICPR 2006, 18th International Conference on Pattern Recognition, vol. 2, pp. 934 - 937, 2006.

8. Woo June Choi, Gihyeon Minj, Beyong Ha Lee, Jonghyun Eom, and Ju Wan Kim, Counterfeit detection using characterization of safety feature on banknote with full-field optical coherence tomography, J. Opt. Soc. Korea, vol. 14. no 4, pp. 316-320, 2010.

9. E. W. Corbett, Not So Funny Funny-Money: The Threat of North Korean Counterfeiting of U.S. Currency, Stevenson University Forensics Journal, vol. 4, pp. 14-22, 2013.

10. L.S. Eberlin et al., Instantaneous chemical profiles of banknotes by ambient mass spectrometry, Analyst, no. 10, pp. 2533-2539, 2010.

11. M. Engineer, Currency transactions costs and competing fiat currencies, J. of Int. Economics, Elsevier, 2000.

12. T. Enomae, Y.-H. Han, A. Isogai, Nondestructive determination of fibre orientation distribution of paper surface by image analysis, Nordic Pulp and Paper Research Journal 21 (2) (2006) 253-259.

13. A. Frosini, M. Gori, and P. Priami, A neural network-based model for paper currency recognition and verification, IEEE transactions on neural networks, vol. 7, no. 6, 1996

14. E. Green, W. Weber, Will the new $\$ 100$ bill decrease counterfeiting?, Federal Reserve Bank of Minneapolis Quarterly Review, vol 20, no. 3, pp. 3-10, 1996.

15. A. Guedes, M. Algarra. A. Carmelo Prieto, B. Valentin, V. Hortelano, S. Neto, R. Algarra, and F. Noronha, Raman microspectroscopy of genuine and fake Euro banknotes, Spectroscopy Lett., An international Journal for Rapid Communication, 46:8, 569-576, 2013.

16. S.K. Nath, and K. Palaniappan, Adaptive robust structure tensors for orientation estimation and image segmentation, Lect. Notes Comput. Sci., 3803, 445-453, 2005.

17. K. Niskanen, Paper physics, papermaking science and technology, vol. 16, Fapet, Jyväskylä, 1998.

18. V. Rusanov, KChakarova, and T.Madolev, Mössbauer spectroscopy investigation of the properties and stability of dollar bank note pigments, Appl. Spectroscopy, vol. 56, no. 9, pp. 1228-1236, 2002.

19. J. Sampo, J. Takalo, S. Siltanen, M. Lassas, A. Miettinen, and J. Timonen, Curvelet-based method for orientation estimation of particles, Optical Engineering, 53(3), 033109, 10 pages, 2014.

20. A. Vila, N. Ferrer, J. Mantocon, D. Breton, and J.F. Garcia, Development of a fast and non-destructive procedure for characterizing and distinguishing original and fake euro notes, Analytica Chimica Acta, vol. 559, no. 2, pp. 257-263, 2006.

21. Chi-Yuan Yeh, Wen-Pin Su, Shie-Jue Lee, Employing multiple-kernel support vector machines for counterfeit banknote recognition, Appl. Soft Comput., vol. 11, no. 1, 1439-1447, 2011. 